\title{
Children's Memory Scale
}

National Cancer Institute

\section{Source}

National Cancer Institute. Children's Memory Scale. NCI Thesaurus. Code C120253.

A test designed to make a comprehensive assessment of learning and memory in

children and adolescents ranging in age from 5 to 16 years. 